SLAC-PUB-13771

CP3-09-45

\title{
Massless versus Kaluza-Klein Gravitons at the LHC
}

\author{
Xavier Calmet 1 a , Priscila de Aquind2 $2, c, c$ and Thomas G. Rizzo $3 d$ \\ a Physics and Astronomy, University of Sussex, \\ Falmer, Brighton, BN1 9QH, UK \\ ${ }^{b}$ Université Catholique de Louvain, CP3, \\ 2, Chemin du Cyclotron, B-1348 Louvain-la-Neuve, Belgium \\ ${ }^{c}$ Katholieke Universiteit Leuven, ITP, \\ Celestijnenlaan 200D - Bus 2415, B-3001 Leuven, Belgium \\ d SLAC National Accelerator Laboratory, \\ 2575 Sand Hill Rd., Menlo Park, CA, 94025, USA
}

\begin{abstract}
We show that the LHC will be able to differentiate between a four-dimensional model with quantum gravity at $\sim 1 \mathrm{TeV}$ where the (massless) graviton becomes strongly coupled to standard model particles at $1 \mathrm{TeV}$ and brane world type models with a large extra-dimensional volume and massive Kaluza-Klein gravitons. We estimate that the $14 \mathrm{TeV}$ LHC could put a limit of the order of $\sim 5 \mathrm{TeV}$ on the four dimensional Planck mass in a model independent way.
\end{abstract}

\footnotetext{
${ }^{1}$ x.calmet@sussex.ac.uk

${ }^{2}$ priscila@itf.fys.kuleuven.be

${ }^{3}$ rizzo@slac.stanford.edu
} 
It is now well understood that the energy scale at which quantum gravitational effects become strong could be much lower than naively estimated using dimensional analysis arguments. For example in models with a brane world and a large extra dimensional volume $[1,2]$ or in four dimensional models with a large hidden sector of some $10^{33}$ particles [3] 4 , the Planck mass could be near $1 \mathrm{TeV}$ and thus in reach of colliders such as the Tevatron or the LHC. In this paper we compare the phenomenology of the extra-dimensional brane world models (i.e., ADD type models) with that of the four-dimensional one. In particular we show that the LHC can differentiate between the two scenarios. Here we follow the strategy developed in [6] to differentiate the phenomenology of Kaluza-Klein gravitons in ADD models from that of unparticles $[7,8]$.

The specific four-dimensional model discussed in [3] is rather different at the theoretical level from the well known ADD model. Gravity can become strong in four dimensions if there is a large hidden sector that only interacts gravitationally with the standard model. This large hidden sector will contribute to the renormalization group running of Newton's constant. If the new particles in this sector have spin 0 and/or $1 / 2$ the running of Newton's constant is such that it becomes significantly larger at lower energy. For $10^{33}$ new particles one gets $M_{P}(1 \mathrm{TeV}) \sim 1 \mathrm{TeV}$. The masses of these new particles determine the energy scale at which the running of Newton's constant starts, for example one could choose $m_{i} \sim 100 \mathrm{GeV}$. In such a case most of the running of the Planck mass (equivalently of Newton's constant) occurs close to $1 \mathrm{TeV}$. One can thus approximate the running of the reduced Planck mass by a step function: for $\mu<1 \mathrm{TeV}, M(\mu)=2.4 \times 10^{18} \mathrm{GeV}$, while for $\mu \geq 1 \mathrm{TeV}, M(\mu) \sim 1$ $\mathrm{TeV}$. The phenomenology of this model is then found to be rather different from that of ADD since strong gravity cuts off at the TeV scale. For example, there are no Kaluza-Klein excitations of the graviton and virtual gravitons will decay massively to the hidden sector and are thus leading to a missing energy signal. It has been suggested in [9] to search for a monojet plus missing energy as a signature of this model at the LHC. This is, however, also one of the familiar smoking gun signals of the ADD scenario and thus it is important to establish that the LHC could differentiate between the two models. Furthermore, in case of non-observation of the monojet + missing energy signals, one could use data from the LHC to place a corresponding lower bound on the value of the Planck mass (i.e. the scale at which quantum gravitational effects become important). It is the aim of the present paper to establish these two points 5

\footnotetext{
${ }^{4}$ See also [4] for another discussion of the value of the Planck mass in models with a large hidden sector. In their approach it is not clear how the value of the Planck mass depends on the spin of the particles in the hidden sector. However, reliable perturbative calculations [5] clearly show a spin dependence: spin 0 and $1 / 2$ particles lower the Planck mass, while spin 1 particles increase it.

${ }^{5}$ Also, unlike in the ADD case, the exchange of these massless gravitons does not lead to effective dim- 8 contact interactions with an associated characteristic TeV scale. Furthermore, there will be no observable
} 
The production of jets with large $E_{T}$ recoiling against a massless graviton $G$ can arise from the parton subprocesses $q+\bar{q} \rightarrow G+g, q+g \rightarrow q+G, \bar{q}+g \rightarrow \bar{q}+G$, and $g+g \rightarrow g+G$ in a manner similar to that in ADD. However, the expressions for the corresponding subprocess cross-sections are different from those found in the ADD model.

The leading order contributions at the parton level for this four dimensional model have been calculated and presented in [9]. The polarization and color averaged cross section for $q+\bar{q} \rightarrow g+G$ is given by

$$
\frac{d \sigma}{d \cos \theta}=\frac{1}{72 \pi} \frac{g_{s}^{2}}{\bar{M}(\mu)^{2}}\left(\frac{s^{2}+2 t^{2}+2 t s}{s^{2}}\right),
$$

where $g_{s}^{2}=4 \pi \alpha_{s}$ is the usual strong coupling constant, hereafter evaluated at the scale of the jet $E_{T}, \bar{M}(\mu)$ is the reduced Planck mass such that Newton's constant is given by $G_{N}=$ $\left(4 \pi \bar{M}^{2}\right)^{-1}$ and where $s$ and $t$ are the subprocess Mandelstam variables: $t=-1 / 2 s(1-\cos \theta)$. The cross sections for $q+g \rightarrow q+G$ and $\bar{q}+g \rightarrow \bar{q}+G$ are given by

$$
\frac{d \sigma}{d \cos \theta}=-\frac{g_{s}^{2}\left(2 s^{2}+2 s t+t^{2}\right)}{192 \pi \bar{M}(\mu)^{2} s t} .
$$

The corresponding matrix element can be obtained using crossing symmetry from that of the transition $q+\bar{q} \rightarrow G+g$. Finally we also obtain the cross section for $g+g \rightarrow g+G$ which is given by

$$
\frac{d \sigma}{d \cos \theta}=-\frac{3 g_{s}^{2}\left(s^{2}+s t+t^{2}\right)^{2}}{128 \pi \bar{M}(\mu)^{2} s^{2} t(s+t)} .
$$

Note that all quarks are treated as massless in these expressions. Recall that these cross sections are essentially zero below the $\sqrt{s}=M_{P}$ threshold. In the case of ADD, the parton level cross-sections have been given in [10] and we quote them here for comparison purposes for KK gravitons of mass $m$. The cross section $q+\bar{q} \rightarrow g+G_{K K}$ where $G_{K K}$ is an ADD Kaluza-Klein graviton is given by:

$$
\begin{aligned}
\frac{d \sigma}{d \cos \theta}\left(q+\bar{q} \rightarrow g+G_{K K}\right)= & \frac{1}{144 \pi} \frac{g_{s}^{2}}{\bar{M}^{2}} \frac{1}{1-m^{2} / s}\left[\left(2-\frac{4 u t}{\left(s-m^{2}\right)^{2}}\right)\left(1+\left(\frac{m^{2}}{s}\right)^{4}\right)\right. \\
& +\left(2 \frac{\left(s-m^{2}\right)^{2}}{4 u t}-5+4 \frac{4 u t}{\left(s-m^{2}\right)^{2}}\right) \frac{m^{2}}{s}\left(1+\left(\frac{m^{2}}{s}\right)^{2}\right)+ \\
& \left.+6\left(\frac{u-t}{s-m^{2}}\right)^{2}\left(\frac{m^{2}}{s}\right)^{2}\right]
\end{aligned}
$$

alteration in Newtonian Gravity observable in table top experiments as may be possible with ADD in the case of 2 extra dimensions. 
where here $s, t, u$ are the Mandelstam variables where we have: $t, u=-1 / 2 s\left(1-m^{2} / s\right)(1 \mp$ $\cos \theta)$. The cross section for $q+g \rightarrow q+G_{K K}$ can be obtained from this expression by crossing $s \leftrightarrow t$ :

$$
\begin{aligned}
\frac{d \sigma}{d \cos \theta}\left(q+g \rightarrow q+G_{K K}\right)= & \frac{g_{s}^{2}}{384 \pi \bar{M}^{2}} \frac{(-t / s)\left(1-m^{2} / s\right)}{\left(1-m^{2} / t\right)^{2}} \times \\
& \times\left[\left(2-\frac{4 u s}{\left(t-m^{2}\right)^{2}}\right)\left(1+\left(\frac{m^{2}}{t}\right)^{4}\right)\right. \\
& +\left(2 \frac{\left(t-m^{2}\right)^{2}}{4 u s}-5+4 \frac{4 u s}{\left(t-m^{2}\right)^{2}}\right) \frac{m^{2}}{t}\left(1+\left(\frac{m^{2}}{t}\right)^{2}\right)+ \\
& \left.+6\left(\frac{s-u}{t-m^{2}}\right)^{2}\left(\frac{m^{2}}{t}\right)^{2}\right] .
\end{aligned}
$$

As in the massless case, the cross section for $\bar{q}+g \rightarrow \bar{q}+G_{K K}$ is also the same as that of $q+g \rightarrow q+G_{K K}$. For the process $g+g \rightarrow g+G_{K K}$, one finds

$$
\begin{aligned}
\frac{d \sigma}{d \cos \theta}\left(g+g \rightarrow g+G_{K K}\right)= & \frac{3}{16} \frac{\pi \alpha_{s} G_{N}}{\left(1-m^{2} / s\right)\left(1-\cos ^{2} \theta\right)} \times \\
& {\left[\left(3+\cos ^{2} \theta\right)^{2}\left(1+\left(\frac{m^{2}}{s}\right)^{4}\right)-4\left(7+\cos ^{4} \theta\right) \frac{m^{2}}{s}\left(1+\left(\frac{m^{2}}{s}\right)^{2}\right)\right.} \\
& \left.+6\left(9-2 \cos ^{2} \theta+\cos ^{4} \theta\right)\left(\frac{m^{2}}{s}\right)^{2}\right] .
\end{aligned}
$$

It is easy to see that one recovers the massless graviton case by taking the mass of the KK graviton to zero. Note that in the ADD case, to obtain the collider cross sections, one has to also perform an integration over the KK-mass distributions as well as the usual integration over the parton densities. Of course in the case of the four dimensional model no KK sum is required. To be specific we will employ the CTEQ6.6 parton distributions [11] in obtaining the results obtained below.

Using the partonic cross sections above we can employ a modified version of the code developed for [6], and can then generate events for the $14 \mathrm{TeV}$ LHC for both the ADD and the four dimensional models as well as for the the standard model background. Here, following Ref. [12], we expect this background to be dominated by the production of $Z$ plus a single jet with the $Z$ decaying into pairs of neutrinos. This background can be much reduced by requiring a missing energy and/or jet energy cut of at least $500 \mathrm{GeV}$ and demanding that the jet to be central $\left|\eta_{j}\right|<3$. The results of our direct comparison of the ADD predictions with those of the four dimensional model assuming, e.g., that $M_{P}=1 \mathrm{TeV}$ can be found in Fig. 1. Here we see that the two new physics models predict monojet $E_{T}$ distributions which are reasonably dissimilar in overall shape. The falling four dimensional model monojet spectrum 
above the $\sqrt{\hat{s}}=M_{P}$ threshold is seen to be somewhat softer than the corresponding ADD model prediction for any number of extra dimensions.

From Figs 2 and 3 we can obtain an estimate of the search reach for the four dimensional model at the $14 \mathrm{TeV} \mathrm{LHC}$ of $\simeq 5 \mathrm{TeV}$ assuming an integrated luminosity of $100 \mathrm{fb}^{-1}$. Here we also see some unusual features in these distributions associated with the four dimensional model which are absent from the case of ADD which are due to the cross section threshold at $\sqrt{\hat{s}}=M_{P}$. Unlike for the ADD case, whose $E_{T}$ and $E_{T}^{c u t}$ distributions fall monotonically, the threshold in the four dimensional model cross section naturally leads to structure in these corresponding distributions. This added kink-like structure is a clear aid in distinguishing the predictions of these two classes of models at the LHC as can easily be seen from these figures. For the case of the $E_{T}^{c u t}$ distribution we see that it is essentially flat until the value $E_{T}^{c u t}=0.5 M_{P}$ is reached and then falls monotonically. On the otherhand, the $E_{T}$ distribution rises below the value of $E_{T}=0.5 M_{P}$ at which point a peak occurs. At higher values of $E_{T}$ the distribution fall monotonically. These are quite distinctive indications of a cutoff in the cross section at a fixed value of $\sqrt{\hat{s}}$ and would allow us to extract this value directly from the LHC experimental data.

Here we have focussed on one aspect of quantum gravity phenomenology at the LHC. Other interesting signatures are small semi-classical black holes [13] or small non-thermal black holes [14]. Although small black holes would be easier to observe than the missing energy signals discussed above, the theoretical framework typically used to discuss the formation of small holes at the LHC is less reliable than the one used here for the emission of gravitons which relies on the well understood expansion of the Hilbert-Einstein action around the Minkowski metric. This approach thus assumes very little about the nature of quantum gravity and the bounds derived that way will therefore be much more reliable than using small black holes.

The LHC will start to operate in the coming months. Although it may take time to reach its design energy and luminosity, it is quite remarkable to realize that once does it will be able to probe not only whether the solution to the hierarchy problem is based on low scale quantum gravity, but it will also give us the possibility to learn whether a low Planck mass is due to large extra-dimensions or a large hidden sector with only four space-time dimensions. The results presented in this work will be useful even if the solution to the gauge hierarchy problem is not based on gravity. In that case, the LHC will be able to set the tightest limit to date on the value of the energy scale at which quantum gravitational effects become strong and this in a model independent way.

Acknowledgments: The work of TGR was supported in part by the Department of Energy, 


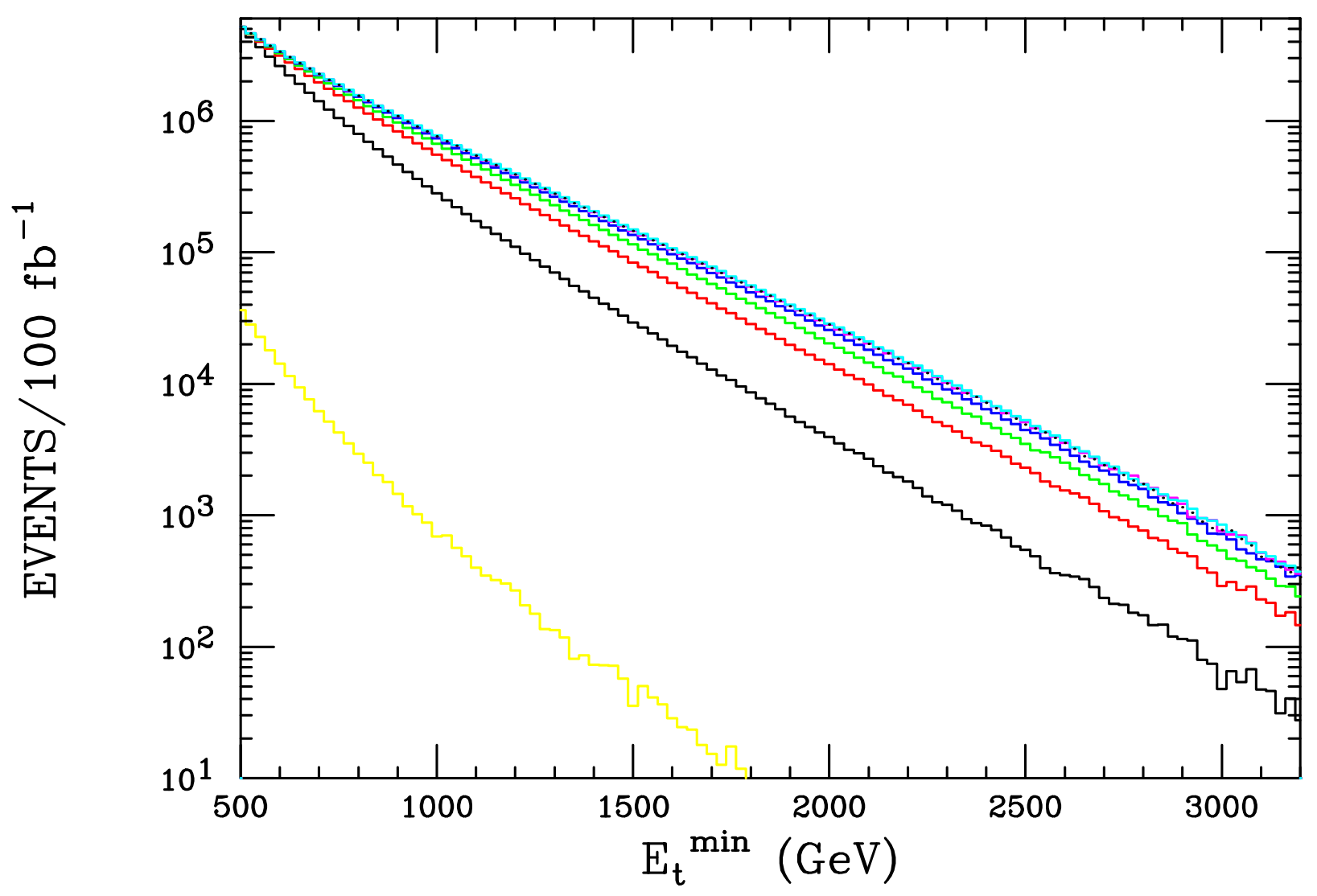

Figure 1: This figure shows the shape of the $E_{T}^{\min }$ distribution counting the number of monojet events at the $14 \mathrm{TeV}$ LHC assuming a luminosity of $100 \mathrm{fb}^{-1}$ above a $E_{T}^{\min }$ cut of $500 \mathrm{GeV}$ and requiring a central jet $\left|\eta_{j}\right|<3$. The yellow histogram is the expected standard model background as discussed in the text while the red and higher histograms are for the ADD model with the number of extra dimensions being 2,3,4, etc. The lower solid black histogram is for the four dimensional model with $M_{P}=1 \mathrm{TeV}$. The ADD results were in each case adjusted by varying their associated Planck scale to produce the same result as does the four dimensional model at $E_{T}^{\min }=500 \mathrm{GeV}$ in order to show the relative shapes for these distributions. 


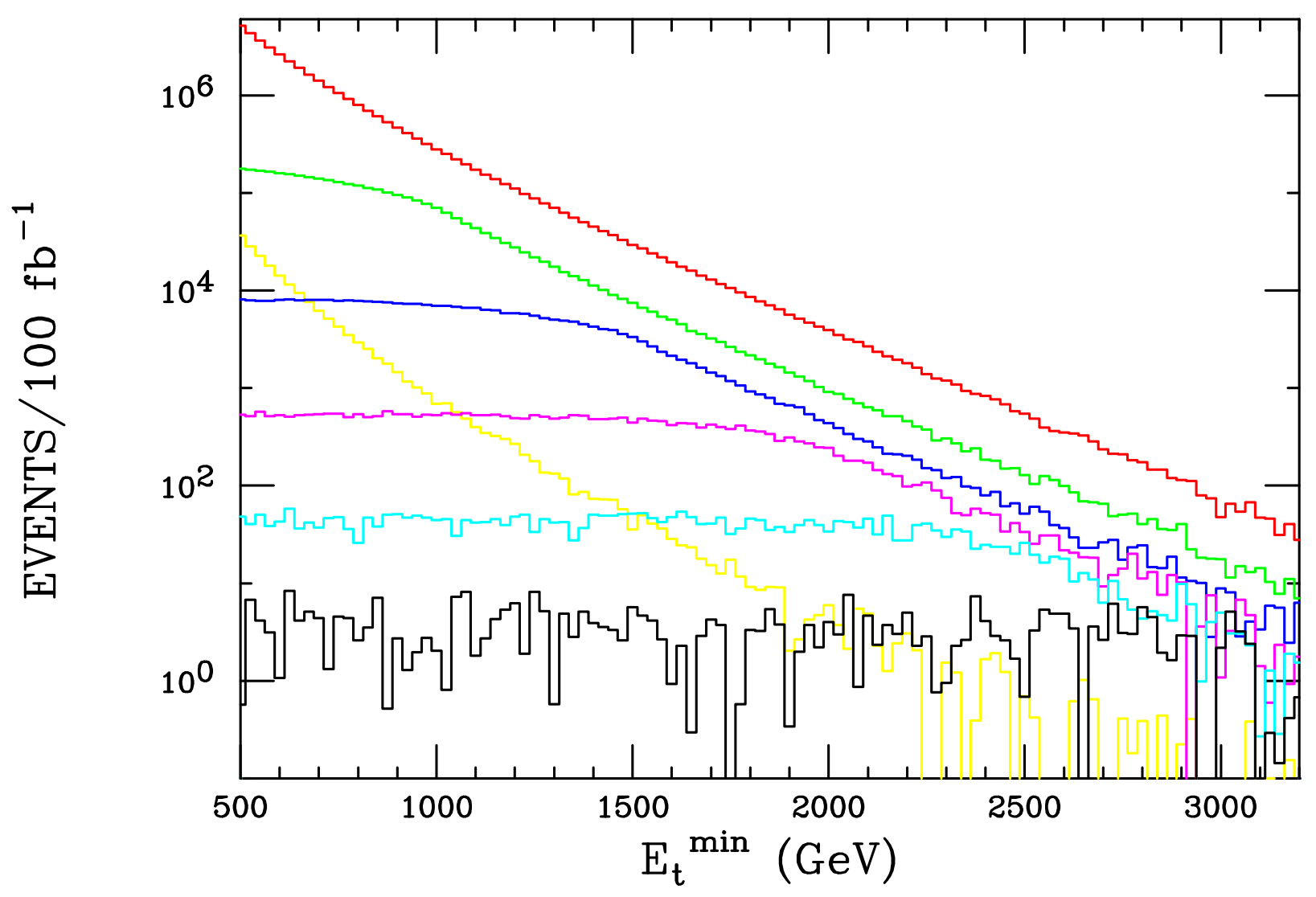

Figure 2: This figure for the $14 \mathrm{TeV}$ LHC shows the event rate for the standard model jet+missing energy background as a function of the cut on the jet $E_{T}$ in yellow as well as the four dimensional model predictions for the cases $M_{P}=1(2,3,4,5,6) \mathrm{TeV}$ from top to bottom in red, green, blue, $\ldots$ and requiring that the existence of a threshold at $\sqrt{\hat{s}}=M_{P}$. From this figure, one can deduce that the search reach for the four dimensional model at the LHC is $\simeq$ $5 \mathrm{TeV}$ for a luminosity $=100 \mathrm{fb}^{-1}$. The shape of the signal histograms with the requirement above are quite different than those for ADD due to the vanishing of the cross section at small $\hat{s}$. Note the shape change at $E_{T}^{\min }=0.5 M_{P}$ which is a result of this cross section threshold that is absent in the ADD model. 


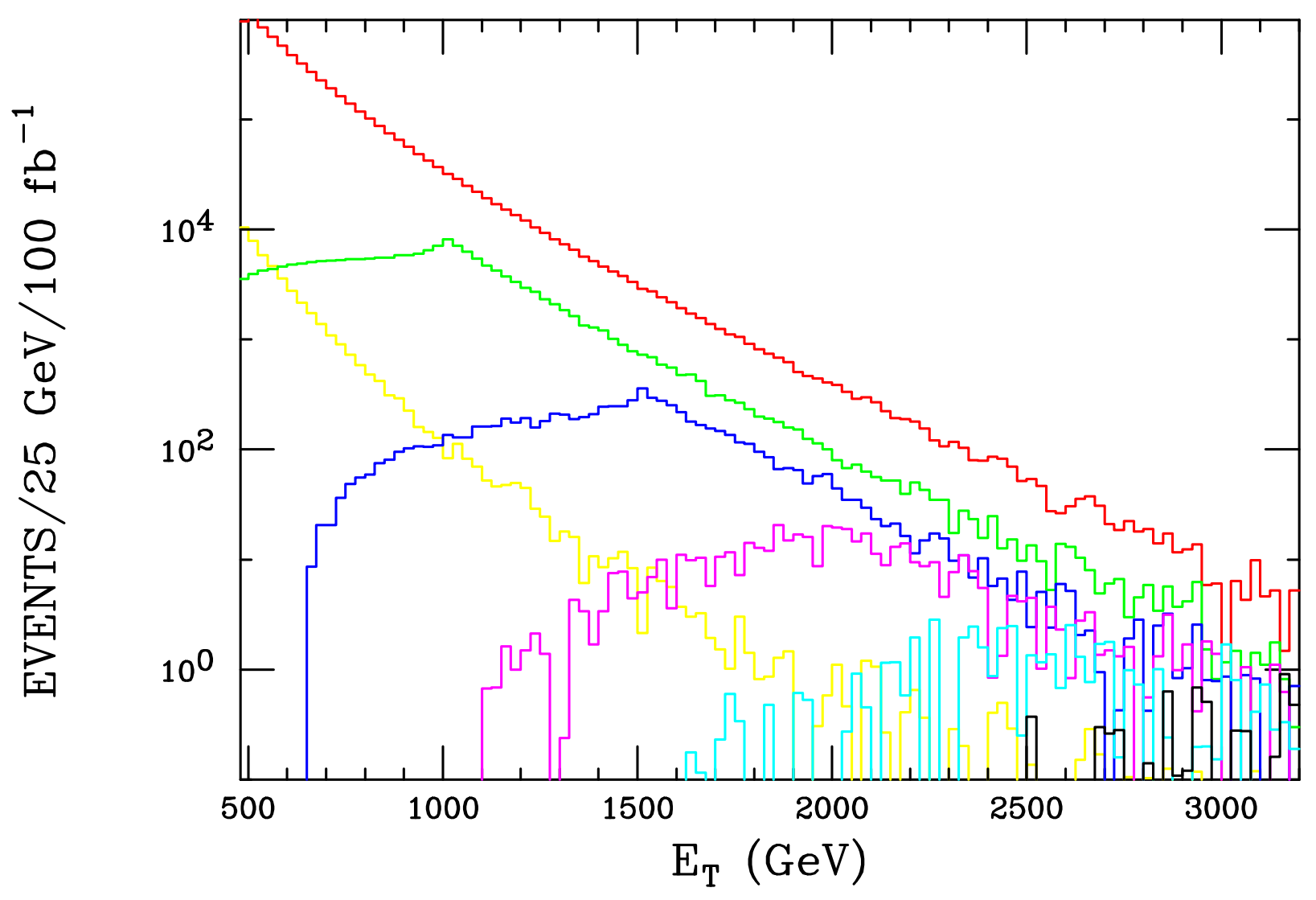

Figure 3: This figure shows the monojet $E_{T}$ distributions at the $14 \mathrm{TeV}$ the LHC assuming a luminosity of $100 \mathrm{fb}^{-1}$. The histograms are as labeled as in the previous figure. Note the kink-like structure in the four dimensional model distributions at $E_{T}=0.5 M_{P}$ which is a result of the cross section threshold that is absent in the ADD model. 
Contract DE-AC02-76SF00515.

\section{References}

[1] N. Arkani-Hamed, S. Dimopoulos and G. R. Dvali, Phys. Lett. B 429, 263 (1998) arXiv:hep-ph/9803315;

[2] L. Randall and R. Sundrum, Phys. Rev. Lett. 83, 3370 (1999) arXiv:hep-ph/9905221.

[3] X. Calmet, S. D. H. Hsu and D. Reeb, Phys. Rev. D 77, 125015 (2008) arXiv:0803.1836 [hep-th]].

[4] G. R. Dvali, G. Gabadadze, M. Kolanovic and F. Nitti, Phys. Rev. D 65, 024031 (2002) arXiv:hep-th/0106058]; G. Dvali, arXiv:0706.2050 [hep-th]; G. Dvali and M. Redi, Phys. Rev. D 77, 045027 (2008) arXiv:0710.4344 [hep-th]].

[5] F. Larsen and F. Wilczek, Nucl. Phys. B 458, 249 (1996) [arXiv:hep-th/9506066.

[6] T. G. Rizzo, JHEP 0811, 039 (2008) [arXiv:0809.4659 [hep-ph]].

[7] H. Georgi, Phys. Lett. B 650, 275 (2007) arXiv:0704.2457 [hep-ph]] and Phys. Rev. Lett. 98, 221601 (2007) arXiv:hep-ph/0703260.

[8] K. Cheung, W. Y. Keung and T. C. Yuan, AIP Conf. Proc. 1078, 156 (2009) arXiv:0809.0995 [hep-ph]].

[9] X. Calmet and P. de Aquino, arXiv:0906.0363 [hep-ph].

[10] E. A. Mirabelli, M. Perelstein and M. E. Peskin, Phys. Rev. Lett. 82, 2236 (1999) arXiv:hep-ph/9811337;

G. F. Giudice, R. Rattazzi and J. D. Wells, Nucl. Phys. B 544, 3 (1999) arXiv:hep-ph/9811291.

[11] P. M. Nadolsky et al., arXiv:0802.0007 [hep-ph].

[12] L. Vacavant and I. Hinchliffe, J. Phys. G 27, 1839 (2001).

[13] S. Dimopoulos and G. L. Landsberg, Phys. Rev. Lett. 87, 161602 (2001) arXiv:hep-ph/0106295.

[14] X. Calmet, W. Gong and S. D. H. Hsu, Phys. Lett. B 668, 20 (2008) arXiv:0806.4605 [hep-ph]]. 